\title{
Healthcare Device Direct Feed Reporting
}

National Cancer Institute

\section{Source}

National Cancer Institute. Healthcare Device Direct Feed Reporting. NCI Thesaurus.

Code C159821.

An indication that vital sign data came directly from a device in a healthcare facility. 